\title{
CHAP-Hyaluronic Acid (CHAP-HA) Filler as an Optimal Candidate for Forehead Filler Augmentation Using a 3-Point Injection Technique
}

\author{
Hsiao-Tung Lee1, Haw-Yueh Thong2* ${ }^{*}$ \\ ${ }^{1}$ Department of Radiology, Shin-Kong Wu Ho-Su Memorial Hospital, Taiwan \\ ${ }^{2}$ Department of Dermatology, Shin-Kong Wu Ho-Su Memorial Hospital, Taiwan \\ Email: ^drkellytang@gmail.com
}

How to cite this paper: Lee, H.-T. and Thong, H.-Y. (2021) CHAP-Hyaluronic Acid (CHAP-HA) Filler as an Optimal Candidate for Forehead Filler Augmentation Using a 3-Point Injection Technique. Journal of Cosmetics, Dermatological Sciences and Applications, 11, 76-83.

https://doi.org/10.4236/jcdsa.2021.112008

Received: March 8, 2021

Accepted: May 29, 2021

Published: June 1, 2021

Copyright $\odot 2021$ by author(s) and Scientific Research Publishing Inc. This work is licensed under the Creative Commons Attribution International License (CC BY 4.0).

http://creativecommons.org/licenses/by/4.0/

(c) (i) Open Access

\begin{abstract}
Minimally invasive forehead augmentation is becoming increasingly popular in Asia. However, treatment of the glabella is associated with injection-related complications. The safety of injections can be increased through a thorough understanding of the anatomy and precise injection planes. The authors propose a 3-point injection technique for forehead augmentation in Asian patients that uses CHAP-hyaluronic acid (CHAP-HA) filler. The longevity of the filler was assessed using high-resolution ultrasound (HRU) imaging. Fifteen patients were examined using HRU before and immediately after treatment and at 1 month, 3 months, 6 months, and 12 months. As evident in HRU imaging, CHAP-HA remained visible within surrounding tissue for $6.4 \pm 3.7$ months. The mean Global Aesthetic Improvement Scale rating was $2.4 \pm 0.5$ immediately after treatment, $2.8 \pm 0.3$ at 1 month, $2.7 \pm 0.5$ at 3 months, $2.1 \pm$ 0.6 at 6 months, and $1.5 \pm 0.6$ at 12 months. Minimal side effects were reported. The authors demonstrated that CHAP-HA filler may be an optimal candidate for forehead filler augmentation using a 3-point injection technique.
\end{abstract}

\section{Keywords}

CHAP-Hyaluronic Acid (CHAP-HA) Filler, High-Resolution Ultrasound (HRU), Forehead Augmentation

\section{Introduction}

The American Society of Plastic Surgeons reported an increasing demand for 
minimally invasive cosmetic procedures in the facial area [1]. Among such procedures, forehead augmentation is increasingly popular in Asia. However, forehead augmentation involving the glabellar region may result in severe complications caused by inadvertent intravascular injection of filler [2] [3]. Knowledge of forehead anatomy and precise injection planes can minimize risk [2] [4]. On review of literature, various materials such as hyaluronic acid (HA) fillers, autologous fat, calcium hydroxyapatite have been used, and various techniques had been proposed [3] [4] [5] [6] [7]. Nonetheless, there is no consensus on injection technique for safe forehead augmentation. For injections into the glabella, Phumyoo et al. suggested a safe technique based on topography, a thorough understanding of arterial distribution, and color Doppler ultrasonographic examination before injection to minimize the risk of severe complications [2]. Kim proposed a novel forehead classification system based on East Asian ethnicity and suggested that first injecting a tumescent solution and then using a minimal number of entry points for the injection of filler reduces the risk of embolization [5].

\section{Aim}

The purpose of this observational study was to determine the safety, clinical tolerability, clinical outcome, and longevity of CHAP-hyaluronic acid (CHAP-HA) filler when using a 3-point injection technique for forehead augmentation. The authors previously demonstrated that high-resolution ultrasound (HRU) allows for the localization of filler injection planes and the study of HA-tissue reactions [8] [9]. In this study, HRU was used to assess the longevity of CHAP-HA in 15 patients followed up for 12 months. The aesthetic outcome and side effects of the patients were recorded. The study found the proposed technique of using durable filler material to be relatively safe and capable of achieving optimal tissue integration in minimally invasive forehead augmentation.

\section{Methods}

\subsection{Material}

The CHAP-HA filler, part of the HYADERMIS product line (SciVision Biotech Inc., Taiwan), is composed of bacterium-derived nonanimal stabilized HA with a homologous particle consistency that uses a cross-linked technology called "CHAP", which accounts for its longevity. Clinically, HYADERMIS Blink is generally injected into the superficial dermis layer to improve fine lines. HYADERMIS Kiss is injected into the middle dermis layer to correct wrinkles or for lip augmentation, and HYADERMIS Smile is injected into the deep dermis for contouring [4] [5]. HYADERMIS Chic is injected into the subcutaneous layer or as deep as the periosteum layer to achieve volumization, contouring, and lifting. In our study, 4 syringes filled with $1 \mathrm{~mL}$ of HYADERMIS Blink were used to perform forehead augmentation in each subject by using a 3-point injection technique. 


\subsection{Patients}

Between August 2019 and December 2020, 15 healthy nonpregnant Asian women who had never received a dermal filler injection or forehead aesthetic treatment (including botulinum toxin injections) were enrolled. Patients with a treatment area with scarring, dermatitis, or open wounds and those with a history of major disease, diabetes mellitus, HIV infection, connective tissue disease, or malignant disease were excluded. Those who had recently (within 3 months) received aesthetic laser/chemical peeling treatment or were using medication that disrupts coagulation (e.g., aspirin, NSAIDs, warfarin) were also excluded. All the patients provided written informed consent. Approval of publication of photographic materials was also obtained. Photographic assessments were made before and immediately after the treatment and at 1 month (visit 1), 3 months (visit 2), 6 months (visit 3), and 12 months (visit 4). Using photographs, physicians at each visit used the Global Aesthetic Improvement Scale (GAIS) to conduct evaluations of the efficacy $(3=$ very much improved, $2=$ much improved, $1=\mathrm{im}$ proved, $0=$ no change, $-1=$ worse). Before and after injection, ultrasound imaging was performed to confirm the depth of injection. HRU visualization (Toshiba Xario), using a 12-5 MHz linear transducer, was performed before and immediately after HA filler injection by a single operator. Images capturing the location, size, and shape of the HA after injection and at 1 month, 3 months, 6 months, and 12 months follow-up were obtained.

\subsection{Preparation and Treatment}

When injecting filler, the distribution of the arteries should be taken into consideration. The vascular relationship between the angular artery, supratrochlear artery, and supraorbital artery form a rich and inconsistent arterial plexus in the glabellar region, and, through their direct connection to the ocular artery, they form a more direct pathway to the central retinal artery [10] [11]. In our study, the forehead was divided into one central compartment and two lateral compartments (temporal areas; Figure 1).

The treatment consisted of the following steps:

1) Tumescent solution (TS) preparation: $0.8 \mathrm{~mL}$ of normal saline was mixed with $0.2 \mathrm{~mL}$ of $2 \%$ lidocaine $\mathrm{HCl}$ with epinephrine 1:50,000 into a solution containing $0.04 \%$ lidocaine with epinephrine 1:250,000.

2) Injection of TS: After antisepsis, a $23 \mathrm{G}$ introducer needle is used to create an entry point. A $23 \mathrm{G}$ or $25 \mathrm{G}$ blunt cannula was then inserted into the puncture site and maneuvered along the natural planes of skin tissue, while consecutively injecting $1 \mathrm{~mL}$ of the TS $(0.5 \mathrm{~mL}$ on each side) into the deep tissue area. The midline entry point was chosen to avoid the glabellar area, and we aimed for it to be $2-2.5 \mathrm{~cm}$ above the horizontal glabellar line (Figure 1(a)) to avoid the potential arteries.

3) Treatment of central compartment (A): Using the same $23 \mathrm{G}$ or $25 \mathrm{G}$ blunt cannula and the same midline entry point (x), a total of $2 \mathrm{~mL}$ of CHAP-HA was 

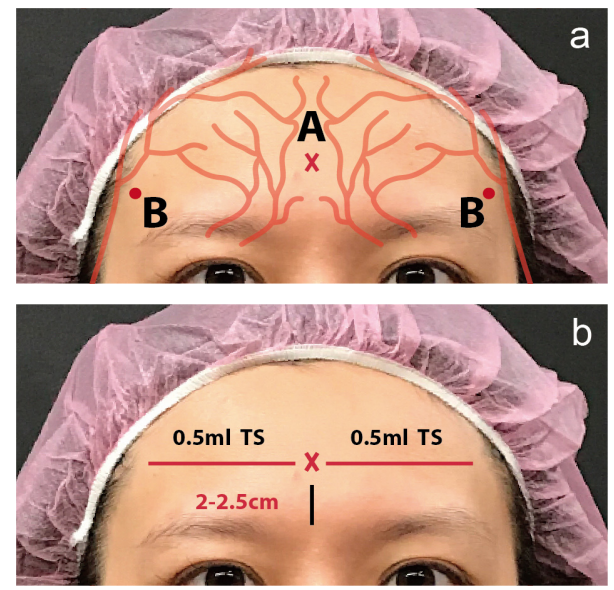

Figure 1. The forehead was categorized into three compartments: (a) central compartment: to be injected with $2 \mathrm{~mL}$ of CHAP-HA, using 23 or $25 \mathrm{G}$ blunt canula with the entry point $(\mathrm{X})$ in the midline after preconditioning with tumescent solution (TS); (b) lateral compartments: to be injected with $0.5 \mathrm{~mL}$ of TS followed by $1 \mathrm{~mL}$ CHAP-HA on each side using $27 \mathrm{G}$ sharp needle then gently massaged to spread the HA material evenly.

injected to the left and right side along the most depressed area of the forehead, adhering to the plane created by the TS, preferably at the supraperiosteal area (sub-Superficial Musculoaponeurotic System; sub-SMAS). Fanning technique and numerous directions of needle advancement were used for even distribution. The injection should be performed slowly and meticulously, avoiding bolus injection, to prevent lumpiness. The filler material was then massaged to evenly distribute it.

4) Treatment of lateral compartments (B): $0.5 \mathrm{~mL}$ of TS was injected via a temple entry point using a $27 \mathrm{G}$ needle. After aspirating and holding for 3 seconds, if no bleeding was observed, the physician injected $1 \mathrm{~mL}$ of CHAP-HA deep (near the temporal bone) on each side using the same $27 \mathrm{G}$ needle, with a bolus injection, and then molded the filler for even distribution.

\section{Results}

In all, 15 patients completed the study (women, mean age $42.1 \pm 8.7$ years). Clinically visible results persisted for up to 12 months (Figure 2). Corresponding HRU imaging assessments were also made (Figure 2). Mean GAIS ratings were $2.4 \pm 0.5$ immediately after treatment, $2.8 \pm 0.3$ at 1 month, $2.7 \pm 0.5$ at 3 months, $2.1 \pm 0.6$ at 6 months, and $1.5 \pm 0.6$ at 12 months (Table 1). Five patients reported experiencing pain during the procedure. After the treatment, 2 patients noted mild bruising, and 10 reported mild swelling; 5 experienced lumpiness (Table 1). All the immediate side effects resolved within a week. Subjects experiencing lumpiness were advised to massage gently the lumpy area daily until the lumpiness became less visible. Most subjects reported that the lumpiness resolved gradually within 1 - 2 weeks. During long-term follow-up visits, all the patients reported natural and cosmetically satisfactory results. No tissue migration or granuloma was noted. 


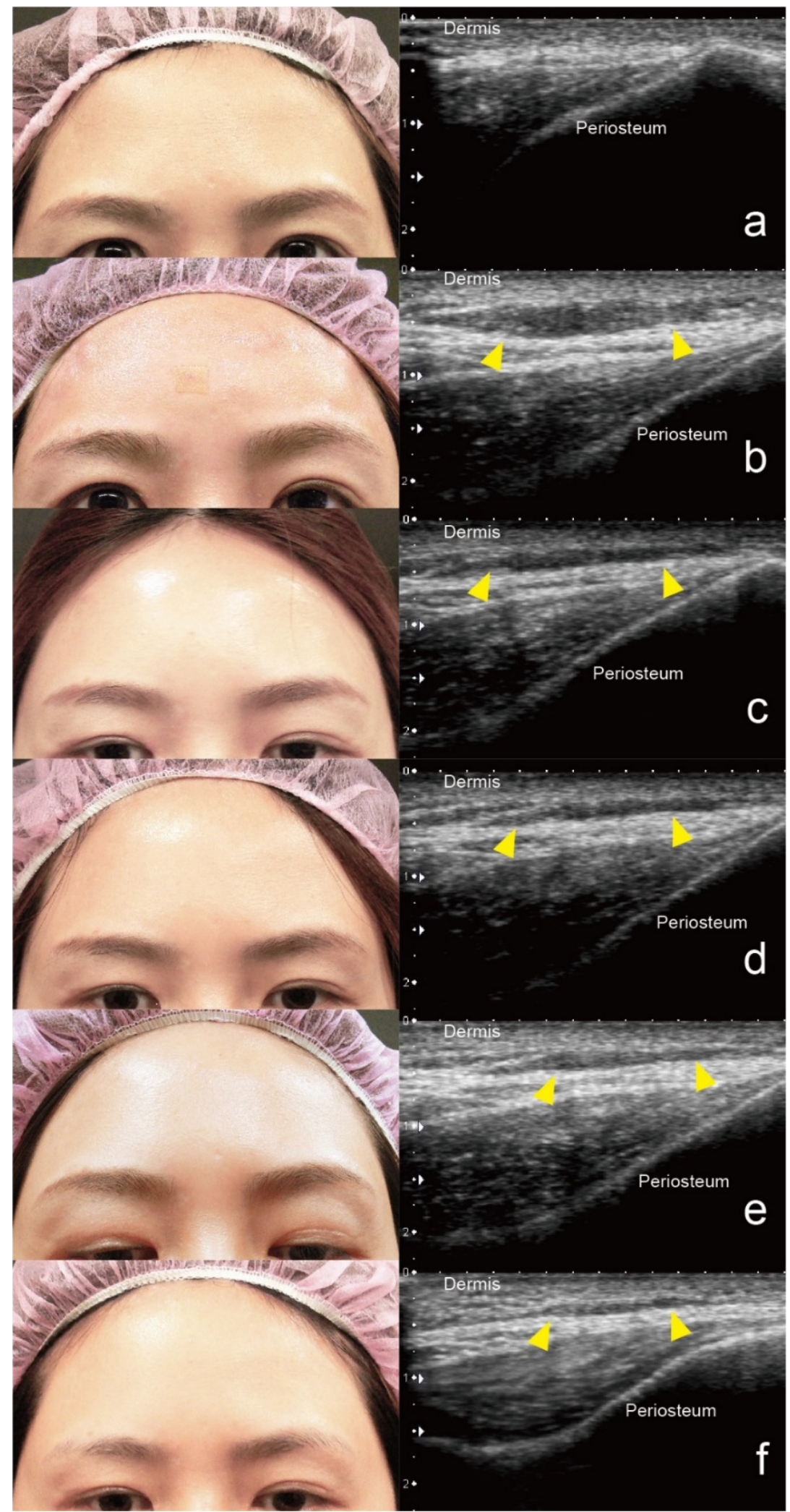

Figure 2. Photographic and HRU imaging assessments (Subject 3). (a) pre-treatment; (b) immediately after; (c) at 1-month follow-up; (d) at 3-month follow-up; (e) at 6-month follow up; (f) at 1-year follow-up visit. The CHAP-HA appeared as hypoechoic densities within the deep tissue plane in HRU (arrows). 
Table 1. GAIS score \& adverse reaction.

\begin{tabular}{|c|c|c|c|c|c|c|c|c|c|c|}
\hline \multirow{2}{*}{ Case No. } & \multirow{2}{*}{ Age } & \multicolumn{5}{|c|}{ Follow up visits (month) } & \multirow{2}{*}{$\begin{array}{l}\text { Pain on } \\
\text { treatment }\end{array}$} & \multirow{2}{*}{$\begin{array}{c}\text { Mean visibility of } \\
\text { CHAP-HA under } \\
\text { HRU imaging }\end{array}$} & \multicolumn{2}{|c|}{ Adverse reaction } \\
\hline & & 0 & 1 & 3 & 6 & 12 & & & Immediate & Delayed ( 1 year) \\
\hline 1 & 37 & 2 & 2 & 3 & 2 & 3 & Acceptable & 6 & Mild swelling & Nil \\
\hline 2 & 30 & 3 & 3 & 3 & 2 & 1 & Acceptable & 6 & Mild swelling & Nil \\
\hline 3 & 35 & 2 & 3 & 3 & 2 & 2 & Acceptable & 12 & Lumpiness & Nil \\
\hline 4 & 32 & 2 & 3 & 3 & 3 & 2 & Acceptable & 12 & Lumpiness & Nil \\
\hline 5 & 32 & 2 & 3 & 3 & 3 & 1 & Mild pain & 6 & Mild swelling & Nil \\
\hline 6 & 50 & 2 & 3 & 3 & 2 & 1 & Acceptable & 3 & Mild swelling & Nil \\
\hline 7 & 42 & 2 & 3 & 3 & 2 & 1 & Mild pain & 3 & $\begin{array}{l}\text { Mild bruising } \\
\text { Mild swelling }\end{array}$ & Nil \\
\hline 8 & 44 & 2 & 2 & 2 & 1 & 1 & Acceptable & 3 & Mild swelling & Nil \\
\hline 9 & 52 & 3 & 3 & 3 & 2 & 2 & Acceptable & 12 & Lumpiness & Nil \\
\hline 10 & 45 & 3 & 3 & 3 & 2 & 1 & Mild pain & 6 & $\begin{array}{l}\text { Mild bruising } \\
\text { Mild swelling }\end{array}$ & Nil \\
\hline 11 & 57 & 3 & 3 & 3 & 3 & 2 & Mild pain & 12 & Lumpiness & Nil \\
\hline 12 & 35 & 2 & 3 & 2 & 2 & 1 & Acceptable & 3 & Mild swelling & Nil \\
\hline 13 & 45 & 2 & 3 & 2 & 2 & 1 & Mild pain & 3 & Mild swelling & Nil \\
\hline 14 & 40 & 3 & 3 & 3 & 2 & 2 & Acceptable & 6 & Lumpiness & Nil \\
\hline 15 & 55 & 3 & 3 & 2 & 1 & 1 & Acceptable & 3 & Mild swelling & Nil \\
\hline Mean & 42.1 & 2.4 & 2.9 & 2.7 & 2.1 & 1.5 & & 6.4 & All resolved within & No nodule nor \\
\hline SD & 8.7 & 0.5 & 0.4 & 0.5 & 0.6 & 0.6 & & 3.7 & one week & granuloma \\
\hline
\end{tabular}

\section{Discussion}

At the level of the glabellar point, the horizontal distances from the midline to the arteries were $4.7 \mathrm{~mm}$ (central artery), $7.8 \mathrm{~mm}$ (paracentral artery), and 14.7 and $19.2 \mathrm{~mm}$ (superficial and deep branches of the supratrochlear artery). The depths from the skin to the arteries were $3.1 \mathrm{~mm}$ (central artery), $4.8 \mathrm{~mm}$ (paracentral artery), and 4.2 and $5.9 \mathrm{~mm}$ (superficial and deep branches of the supratrochlear artery) [2]. Various techniques have been proposed to ensure safety in administering treatments to the forehead region [2] [4] [5] [10] [11] [12]. Kim proposed that minimizing the number of entry points and physical proximity to vessels and nerves during injection reduces risks of embolization and bleeding [5]. Beleznay et al. proposed that injections in the upper forehead be made under a periosteal plane to avoid intravascular cannulation because the supratrochlear artery at this point takes its place at the subcutaneous plane [13]. In our tech- 
nique, the choice of our midline entry point, preconditioning with TS, and deep injections using a blunt canula avoided a direct approach to the glabellar area and hence greatly reduced the risk of vascular complications that commonly occur when treating this area.

Various materials have been proposed for use in minimally invasive forehead augmentation, such as autologous fat, HA fillers, calcium hydroxyapatite, and combinations of these [2] [5] [6] [7]. In our study, CHAP-HA Blink was selected for its low G' and viscosity, superior moldability, and shape retention capacity. Our study determined it was optimal for soft tissue augmentation in the forehead region. HRU imaging assessment after injections revealed optimal tissue integration, which correlated well with clinical satisfaction.

Traditional methods such as using HA filler may be associated with migration and lumpiness; however, in our study, CHAP filler was easy to deliver, molded naturally to native contours, and mimicked normal textures with minimal lumpiness and satisfactory longevity, as was apparent in HRU imaging. The 3-injection-point technique entails minimal risk, exhibits good clinical tolerability, and achieves positive patient outcomes. Preconditioning with TS and using the midline entry point and deep injections delivered via a blunt canula reduced the risk of vessel compromise. The cosmetic results were satisfactory.

\section{Conclusion}

Three-point injection of filler in the forehead region is a technique that is relatively safe, rapid, and easy to perform, involves little down time, and can result in a high rate of patient satisfaction. CHAP-HA filler is a suitable candidate material with satisfactory aesthetic outcomes in forehead augmentation.

\section{Disclosures}

The authors have no conflict of interest to disclose. There are no funding sources for this work.

\section{References}

[1] American Society of Plastic Surgeons (2019) 2019 and 2018 Plastic Surgery Statistics Report. https://www.plasticsurgery.org/news/plastic-surgery-statistics

[2] Phumyoo, T., Jiirasutat, N., Jitaree, B., Rungsawang, C., Uruwan, S. and Tansatit, T. (2020) Anatomical and Ultrasonography-Based Investigation to Localize the Arteries on the Central Forehead Region During the Glabellar Augmentation Procedure. Clinical Anatomy, 33, 370-382. https://doi.org/10.1002/ca.23516

[3] Liu, H., Chen, D. and Zhang, J. (2020) Ophthalmic Artery Occlusion after Forehead Autologous Fat Injection. Retinal Cases and Brief Reports, 14, 271-274. https://doi.org/10.1097/ICB.0000000000000694

[4] Hong, W.J., Liao, Z.F., Zeng, L., Luo, C.E. and Luo, S.K. (2020) Tomography of the Forehead Arteries and Tailored Filler Injection for Forehead Volumizing and Contouring. Dermatologic Surgery, 46, 1615-1620. https://doi.org/10.1097/DSS.0000000000002561

[5] Kim, J. (2018) Novel Forehead Augmentation Strategy: Forehead Depression Cate- 
gorization and Calcium-Hydroxyapatite Filler Delivery after Tumescent Injection. Plastic and Reconstructive Surgery-Global Open, 6, e1858. https://doi.org/10.1097/GOX.0000000000001858

[6] Chao, Y. (2018) Saline Hydrodissection: A Novel Technique for the Injection of Calcium Hydroxylapatite Fillers in the Forehead. Dermatologic Surgery, 44, 133-136. https://doi.org/10.1097/DSS.0000000000001136

[7] Dubina, M., Tung, R., Bolotin, D., Mahoney, A.M., Tayebi, B., Sato, M., Mulinari-Brenner, F., Jones, T., West, D.P., Poon, E., Nodzenski, M. and Alam, M. (2013) Treatment of Forehead/Glabellar Rhytide Complex with Combination Botulinum Toxin A and Hyaluronic Acid Versus Botulinum Toxin A Injection Alone: A Split-Face, Rater-Blinded, Randomized Control Trial. Journal of Cosmetic Dermatology, 12, 261-266. https://doi.org/10.1111/jocd.12059

[8] Lee, H.T. and Thong, H.Y. (2018) Use of High-Resolution Ultrasound (HRU) in the Assessment of Deep Injections of CHAP-Hyaluronic Acid (CHAP-HA) Fillers for Midface Lift. Journal of Cosmetics, Dermatological Sciences and Applications, 08, 126-132. https://doi.org/10.4236/jcdsa.2018.83014

[9] Liang, C.P. and Thong, H.Y. (2016) A Guide to Cheek Augmentation: Single-Point Deep Injection of Hyaluronic Acid Filler at Midface in Close Proximity to Medial Suborbicularis Oculi Fat (SOOF) Area. Journal of Cosmetics, Dermatological Sciences and Applications, 6, 1-8. https://doi.org/10.4236/jcdsa.2016.61001

[10] Cho, K.H., Dalla Pozza, E., Toth, G., Bassiri Gharb, B. and Zins, J.E. (2019) Pathophysiology Study of Filler Induced Blindness. Aesthetic Surgery Journal, 39, 96-106. https://doi.org/10.1093/asj/sjy141

[11] Wu, S., Pan, L., Wu, H., Shi, H., Zhao, Y., Ji, Y. and Zeng, H. (2017) Anatomic Study of Ophthalmic Artery Embolism Following Cosmetic Injection. Journal of Craniofacial Surgery, 28, 1578-1581. https://doi.org/10.1097/SCS.0000000000003674

[12] Scheuer, J.F., Sieber, D.A., Pezeshk, R.A., Gassman, A.A., Campbell, C.F. and Rohrich, R.J. (2017) Facial Danger Zones: Techniques to Maximize Safety During Soft-Tissue Filler Injections. Plastic and Reconstructive Surgery, 139, 1103-1108. https://doi.org/10.1097/PRS.0000000000003309

[13] Beleznay, K., Carruthers, J.D., Humphrey, S. and Jones, D. (2015) Avoiding and Treating Blindness from Fillers: A Review of the World Literature. Dermatologic Surgery, 41, 1097-1117. https://doi.org/10.1097/DSS.0000000000000486 